\title{
miR-339-5p inhibits metastasis of non-small cell lung cancer by regulating the epithelial-to-mesenchymal transition
}

\author{
YUN LI ${ }^{1,2^{*}}$, XUELIN ZHANG ${ }^{1 *}$, ZHEN YANG $^{1}$, YANAN LI $^{3}$, BAIYU HAN $^{4}$ and LIANG AN CHEN ${ }^{1}$ \\ ${ }^{1}$ Department of Respiratory Medicine, Chinese People's Liberation Army (PLA) General Hospital and \\ Chinese PLA Medical School, Beijing 100853; ${ }^{2}$ Department of Respiratory Medicine, The 309th Hospital of \\ Chinese PLA, Beijing 100091; ${ }^{3}$ Department of Internal Medicine, Beijing Aerospace General Hospital, Beijing 100076; \\ ${ }^{4}$ Department of Endocrinology and Metabolism, The 264th Hospital of PLA, Taiyaun, Shanxi 030000, P.R. China
}

Received November 10, 2015; Accepted May 5, 2017

DOI: $10.3892 / 01.2017 .7608$

\begin{abstract}
Metastasis is a common event in cancer pathology, and represents the primary cause of cancer-associated mortality. Metastasis, which is the process in which cancer cells at the primary tumor site spread to a different location in the body and form a new tumor, is regulated by multiple factors and includes a number of steps and stages. In our previous study, it was demonstrated miR-339-5p inhibits cell migration and invasion in vitro and is associated with the tumor-node-metastasis stage and the lymph node metastasis status of non-small cell lung cancer. In the present study, expression of miR-339-5p was first determined in the tissues and peripheral blood of patients with non-small cell lung cancer (NSCLC) and in NSCLC cell lines. It was then demonstrated that miR-339-5p inhibits A549 and H1299 cell invasion. The underlying molecular events of miR-339-5p action in NSCLC were also explored. By luciferase assay and western blot analysis, B-cell CLL/lymphoma 6 (BCL6) was verified as the direct target gene of miR-339-5p. miR-339-5p may inhibit lung cancer cell invasion and migration by regulating the epithelial-to-mesenchymal transition via BCL6 in vitro. It was also demonstrated that the relative expression of miR-339-5p in the peripheral blood is associated with cancer metastasis in patients with non-small cell lung cancer.
\end{abstract}

\section{Introduction}

Lung cancer accounts for $\sim 14 \%$ of incident cases of all cancers, and was the most common cause of cancer-associated

Correspondence to: Professor Liang An Chen, Department of Respiratory Medicine, Chinese People's Liberation Army (PLA) General Hospital and Chinese PLA Medical School, 28 Fuxing Road, Haidian, Beijing 100853, P.R. China

E-mail: yunli_309@163.com

*Contributed equally

Key words: microRNA, metastasis, non-small cell lung cancer, B-cell CLL/lymphoma 6, epithelial-to-mesenchymal transition mortality in the USA in 2013 (1). Metastasis is the process in which cancer cells in the primary site spread to a different location in the body and form a new tumor; this involves entry of tumor cells into circulatory systems (blood and lymph) from the original site, attachment to distant organs, cloning and angiogenesis (2). Metastasis is a common event in cancer pathology, and represents the primary cause of cancer-associated mortality. In lung cancer, metastasis is regulated by numerous factors and involves a number of steps and stages (1-3). Improved understanding of the molecular mechanisms of metastasis in lung cancer may lead to more effective clinical control of lung cancer.

MicroRNAs (miRNAs/miRs) are a class of endogenous, non-coding, single-stranded RNAs with a length of 20-25 nucleotides. By interacting with the 3'-untranslated regions (3'-UTRs) of target mRNAs, miRNAs silence protein expression post-transcriptionally in two ways: Degradation of the mRNAs, or inhibition of mRNA translation into proteins. miRNAs serve important roles in numerous cellular processes, including proliferation, differentiation and apoptosis (4-7), and in the development and progression of lung cancer (8-10).

Our previous study (11) identified that miR-339-5p was downregulated in non-small cell lung cancer (NSCLC) cells and tissue samples, and that its expression was inversely associated with tumor-node-metastasis (TNM) stage. Furthermore, its upregulation could inhibit NSCLC cell migration and invasion in vitro. Bioinformatics analyses also demonstrated that the B-cell CLL/lymphoma 6 (BCL6) and valosin-containing protein genes may be potential targets of miR-339-5p (11). The present study aimed to confirm that BCL6 is the target gene of miR-339-5p, and that miR-339-5p represses the epithelial-to-mesenchymal transition (EMT) of NSCLC cells via BCL6 regulation. EMTs are vital for morphogenesis during embryonic development and are also implicated in the conversion of early stage tumors into invasive malignancies $(2,12)$. During the EMT process, epithelial tumor cells lose their expression of E-cadherin, which is an important epithelial cell-cell adhesion molecule, and begin to exhibit motility and invasiveness (12). In addition, the relative expression of miR-339-5p in peripheral blood and its association with disease progression were investigated. 


\section{Materials and methods}

Cell culture. The NSCLC A549, H1299, H810, H358 and H1975 cell lines were provided by the Department of Respiratory Diseases (purchased from the American Type Culture Collection and maintained at the Chinese People's Liberation Army General Hospital, Beijing, China). These cells were grown in RPMI-1640 medium containing $10 \%$ fetal bovine serum (both from Gibco; Thermo Fisher Scientific, Inc., Waltham, MA, USA), in a humidified atmosphere of $95 \%$ air and $5 \% \mathrm{CO}_{2}$ at $37^{\circ} \mathrm{C}$.

NSCLC tissue specimensand peripheral blood. The present study was approved by the Ethics Committee of the 309th Hospital of Chinese PLA (Beijing, China). A total of 60 surgical NSCLC tissue specimens or biopsy specimens and pairing peripheral blood samples were obtained from the 309th Hospital of Chinese PLA. The baseline clinical features of the patient are shown in Table I.

All patients signed informed consent forms. A total of 32 patients underwent complete surgical resection of the primary tumor. Following surgical resection or biopsy, the peripheral blood samples were obtained. After 6 months, the peripheral blood samples were obtained again. Patients who had received chemotherapy or radiation therapy prior to surgery, or who had rheumatic disease, acute infection, human immunodeficiency virus infection or other types of cancer, were excluded from the present study. The clinical staging was performed according to 7th American Joint Committee on Cancer/Union for International Cancer Control TNM classification (13).

Isolation of total $R N A$ and reverse transcription $(R T)$-quantitative polymerase chain reaction ( $q P C R)$ analysis. RNA from the NSCLC cells and fresh tissue samples was extracted using the mirVana ${ }^{\mathrm{TM}}$ RNA Isolation kit (catalog no. AM1560; Ambion $^{\circledR}$; Thermo Fisher Scientific, Inc.) according to the manufacturer's protocol. RNA from peripheral blood cells was isolated with the miRNeasy ${ }^{\mathrm{TM}}$ Mini kit (catalog no. 217004; Qiagen, Inc., Valencia, CA, USA). The RNA quality and quantity were determined by spectrophotometry (ND-1000; NanoDrop Technologies; Thermo Fisher Scientific, Inc.).

RNA samples from lung cancer cells, tissue samples or peripheral blood cells were subjected to RT reactions using the TaqMan MicroRNA Reverse Transcription kit (catalog no. 4366596; Thermo Fisher Scientific, Inc.). Primers were: miRNA-339-5p (catalog no. 4427975-ID002257) and U6, (catalog no. 44279975-ID001973) (Thermo Fisher Scientific, Inc.). The thermocycling conditions were as follows: $16^{\circ} \mathrm{C}$ for $30 \mathrm{~min}$ and $42^{\circ} \mathrm{C}$ for $30 \mathrm{~min}, 85^{\circ} \mathrm{C}$ for $5 \mathrm{~min}$ and $4^{\circ} \mathrm{C}$ for $5 \mathrm{~min}$. The cDNA was then amplified by qPCR using TaqMan assays with the aforementioned primers and TaqMan Universal Master Mix (catalog no. 4440040; Thermo Fisher Scientific, Inc.) in three independent experiments according to the manufacturer's protocol. The thermocycling conditions were as follows: $50^{\circ} \mathrm{C}$ for $2 \mathrm{~min}$ and $95^{\circ} \mathrm{C}$ for $10 \mathrm{~min}$, for 40 cycles, and each cycle contained two steps, $95^{\circ} \mathrm{C}$ for $15 \mathrm{sec}$, then $60^{\circ} \mathrm{C}$ for $60 \mathrm{sec}$ ). PCR was performed in triplicate for each individual sample. U6 small nuclear RNA was used as an internal
Table I. Baseline clinical features of patients.

\begin{tabular}{|c|c|}
\hline Clinical features & Case no \\
\hline \multicolumn{2}{|l|}{ Age (years) } \\
\hline$\geq 60$ & 41 \\
\hline$<60$ & 19 \\
\hline \multicolumn{2}{|l|}{ Gender } \\
\hline Female & 31 \\
\hline Male & 29 \\
\hline \multicolumn{2}{|l|}{ Smoking history } \\
\hline Yes & 21 \\
\hline No & 39 \\
\hline \multicolumn{2}{|l|}{ Pathological type } \\
\hline Adenocarcinoma & 41 \\
\hline Squamous cell carcinoma & 10 \\
\hline Mixed type & 9 \\
\hline \multicolumn{2}{|l|}{ Clinical stage } \\
\hline I & 10 \\
\hline II & 17 \\
\hline III & 21 \\
\hline IV & 12 \\
\hline \multicolumn{2}{|l|}{ Lymph node metastases } \\
\hline No & 12 \\
\hline N1 & 11 \\
\hline $\mathrm{N} 2$ & 7 \\
\hline N3 & 30 \\
\hline \multicolumn{2}{|l|}{ Distant metastases } \\
\hline M0 & 38 \\
\hline M1 & 12 \\
\hline
\end{tabular}

control. The miRNA expression data are presented as the fold difference relative to U6 based on the $2^{-\Delta \Delta \mathrm{Cq}}$ method (14), where $\Delta \mathrm{Cq}=$ CqmiRNA-CqU6.

Transient miRNA transfection. A549 and H1299 cells $\left(1 \times 10^{6}\right)$ were seeded and cultured overnight in 6-well plates. Subsequently, the cells were transfected with 20 pmol miR-339-5p mimic (Shanghai GenePharma Co., Ltd., Shanghai, China) or 20 pmol 2'-O methylated single-stranded miR-339-5p antisense oligonucleotides (inhibitor; Shanghai GenePharma Co.,Ltd.) using Lipofectamine $2000^{\circledR}$ (Invitrogen; Thermo Fisher Scientific, Inc.) according to the manufacturer's protocol. The miRNA mimics consisted of small double-stranded RNA oligonucleotides with the following sequence: positive-sense strand, 5'-UCCCUGUCCUCCAGG AGCUCACG-3'; and antisense strand, 5'-UGAGCUCCUGGA GGACAGGGAUU-3'. The inhibitor sequence was 5'-CGU GAGCUCCUGGAGGACAGGGA-3'. Negative control RNAs (Shanghai GenePharma Co., Ltd.) were used to eliminate potential nonsequence-specific effects, and the sequences used were not homologous to any sequence in the human genome. The sequences were, 5'-UUCUCCGAACGUGUCACGUTT-3' (positive-sense strand) and 5'-ACGUGACACGUUCGUAGA ATT-3' (antisense strand; a negative control for the miRNA 
mimic) and 5'-CAGUACUUUUGUGUAGUACAA-3' (a negative control for the mRNA antisense transfection).

Cell invasion assay. Transwell inserts (24-well insert; pore size, $8 \mu \mathrm{m}$; Corning Incorporated, Corning, NY, USA) were used to explore the effects of miR-339-5p on A549 and H1299 cell invasion in vitro. The inserts were initially coated with $100 \mu \mathrm{l}$ pre-cooled serum-free RPMI-1640 and Matrigel (1:10; BD Biosciences, Franklin Lakes, NJ, USA) and allowed to solidify at $37^{\circ} \mathrm{C}$ in $5 \% \mathrm{CO}_{2}$ for $3 \mathrm{~h}$. Subsequently, $5 \times 10^{4}$ cells were resuspended in $100 \mu 1$ erum-free RPMI-1640 and placed in the top portion of each Transwell chamber. The lower chamber was filled with RPMI-1640 medium containing 10\% fetal calf serum (HyClone; GE Healthcare Life Sciences, Little Chalfont, UK) as a chemoattractant, and the plates were then incubated at $37^{\circ} \mathrm{C}$ in $5 \% \mathrm{CO}_{2}$ for $24 \mathrm{~h}$. The cells on the upper surface of the membrane were removed using cotton swabs and PBS, and the cells on the lower surface of the insert were fixed in $75 \%$ methanol and stained with $0.1 \%$ crystal violet at room temperature for $15 \mathrm{~min}$. Images of fiverandom fields of view for each insert were captured under a light microscope at x200 magnification. The cells in the images were counted, and the data were summarized as the mean \pm standard deviation. The assays were conducted in duplicate for three independent experiments.

Plasmid construction. The BCL6 3'-UTR was amplified with the following primers: $B C L 6-3^{\prime}-\mathrm{UTR}-$ long non-coding (lnc)-up, 5'-CGGAATTCAGCATGGAGTGTTGATGCTTTC GTCTCCA-3'; and BCL6-3'-UTR-lnc-down, 5'-GCTCTAGAC CAGTTTGACTTTTCA ACATTTTATTCTTATA-3'. The PCR product was inserted between the $X b a \mathrm{I}$ and EcoRI sites, immediately downstream of the stop codon of luciferase gene in pGL3M, which was obtained from the pGL3-control vector (Promega Corporation, Madison, WI, USA) by inserting PstI, $E c o$ RI and NdeI sites (15). To introduce mutations into the seed sequences in the BCL6 3'-UTR, recombinant PCR was performed using the aforementioned primers and the following primers: BCL6-3'-UTR mutated (mut)-down, 5'-AAAGTG ATCCGGAGTAGTTATAACACAAGCATGACGCAGA-3'; BCL6-3'-UTR mut-up, 5'-GCGTCATGCTTGTGTTATAAC TACTCCGCATCACTTTTTT-3'. The mut-down and mut-up primers were respectively used with lnc-up and lnc-down primers for two PCR. Then the two products were purified and mixed. Subsequently, lnc-up and lnc-down primers were used to amplify the mixture. Finally, the mutation for the seed sequence was gained.

Luciferase reporter assay. pGL3-BCL6 3'-UTR and pGL3-BCL6 3'-UTR-mut were co-transfected with 20 pmol miRNA-339-5p mimics or negative controls into A549 and H1299 cells using Lipofectamine ${ }^{\circledR} 2000$ (Invitrogen; Thermo Fisher Scientific, Inc.). Luciferase assay (Promega Corporation) was performed by detecting firefly luciferase activity with normalizing to Renilla luciferase activity after $48 \mathrm{~h}$ of transfection as previous study (16). All experiments were performed in triplicate and repeated three times.

Western blot analysis. Following transfection of the cells with miR-339-5P mimics, inhibitors or controls, western blot analyses were performed, as previously described (15), to detect the levels of BCL6, as well as the EMT-associated markers neural $(\mathrm{N})$-cadherin and epithelial $(\mathrm{E})$-cadherin, using GAPDH as the loading control. The primary antibodies were BCL-6 (D-8; catalog no. sc-7388), GAPDH (0411; catalog no. sc-47724) and NFkBp65 (C-20; catalog no. sc-372) (all from Santa Cruz Biotechnology, Inc., Dallas, TX, USA. Each experiment was repeated three times.

Recovery of BCL6 expression. The expression vector for the BCL6 gene was generated by cloning the PCR product into a pGL3M vector using the following primers: 5'-CGGAAT TCAGGCATTGGACTCAAGATGG-3'; and 5'-GCTCTA GAAACAAAAACATTCCCCCACA-3'. For transfection, cells were seeded in 6-well plates and then transfected with the indicated plasmids using Lipofectamine 2000 (Invitrogen; Thermo Fisher Scientific, Inc.) according to the manufacturer's protocol.

Statistical analysis. All statistical analyses were performed using SPSS v13.0 (SPSS, Inc., Chicago, IL, USA). Differences between variables were assessed by Student's t-test. A Spearman's correlation analysis was used to determine the correlation between miR-339-5p expression levels in the tissues and in the paired peripheral blood samples. $\mathrm{P}<0.05$ was considered to indicate a statistically significant difference.

\section{Results}

miR-339-5p expression in NSCLC cells and patients with NSCLC. To verify the expression of miR-339-5p in NSCLC, five typical NSCLC cell lines were used. As demonstrated in Fig. 1A, miR-339-5p was expressed in all of the cells, and its expression was highest in A549 cells and lowest in H1299 cells; these two cells lines were then used for further study.

The levels of miR-339-5p were also measured in the peripheral blood samples of 60 patients at the time of their admission to hospital and 6 months later, in order to determine the association between the expression of miR-339-5p in peripheral blood cells and NSCLC metastasis. Spearman's correlation analysis demonstrated that miR-339-5p levels in peripheral blood samples were positively correlated to the levels in tumor tissues $(r=0.932$; Fig. 1B), indicating that peripheral miR-339-5p was a good marker for additional study. At 6 months after admission, the peripheral miR-339-5p expression levels in the patients $(n=27)$ with non-new metastatic cancer was not significantly different from that at the time of admission ( $\mathrm{P}>0.05$; Fig. 1C). Conversely, peripheral miR-339-5p expression in the patients $(n=33)$ with new metastatic cancer was increased significantly after 6 months $(\mathrm{P}<0.05$; Fig. 1D) compared with that at the time of hospital admission.

miR-339-5p inhibits A549 and H1299 cell invasion. A gain-of-function assay was adopted in A549 and H1299 cell lines to reveal the effect of miR-339-5p on invasion, by transfecting miR-339-5p mimics into the two cell lines. RT-qPCR confirmed a significant increase in the expression of miR-339-5p in cells transfected with the mimic compared with cells transfected with the negative control, and this increase was concomitant with a significant decrease in the number of 

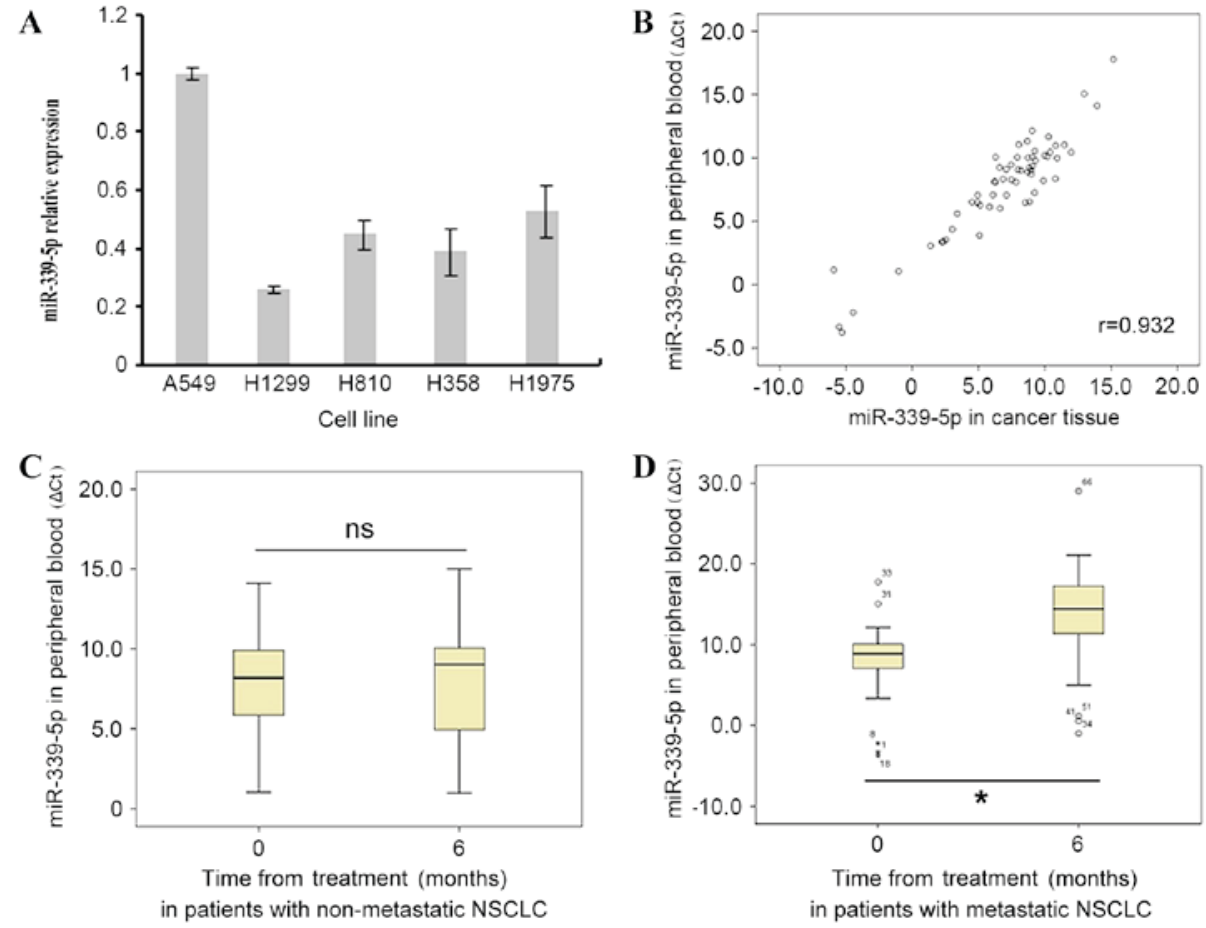

Figure 1. Expression of miR-339-5p in five NSCLC cell lines and in patients with NSCLC. (A) Relativeexpression of miR-339-5p in the NSCLC cell lines A549, H1299, H810, H358 and H1975 (the relative expression of A549 is the reference). (B) miR-339-5p expression levels $(\Delta$ Cq) were determined to be positively correlated $(\mathrm{r}=0.932)$ between cancer tissues and peripheral blood samples in patients with NSCLC, as assessed by Spearman's rank correlation analysis. (C and D) Peripheral miR-339-5p expression $(\Delta \mathrm{Cq})$ levels were determined at different time points relative to treatment $(0$ represents the time of first obtaining tissue samples) of patients with (C) non-metastatic cancer ( $n=27)$ and (D) metastatic cancer ( $n=33)$ ("P<0.05; ns, not significant). miR, microRNA; NSCLC, non-small cell lung cancer.
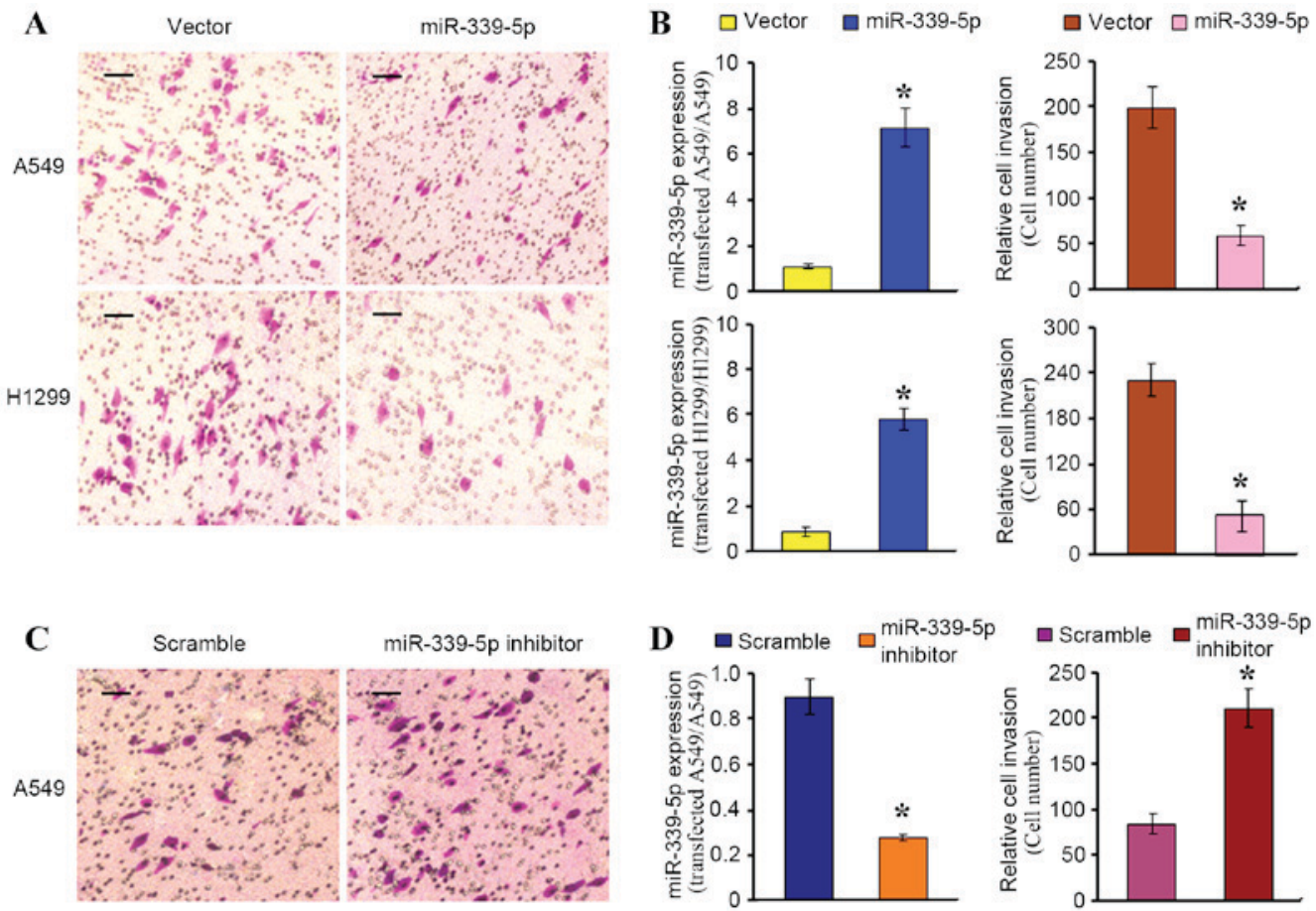

Figure 2. Inhibition of cell invasion by miR-339-5p in non-small cell lung cancer cells. A549 or H1299 cells were transfected with (A and B) miR-339-5p mimics or (C and D) miR-339-5p inhibitors, or their respective controls. Cell invasion was evaluated using Matrigel-coated Transwell chambers, and invaded cells were fixed and stained with $0.1 \%$ crystal violet. (A and C) Representative images of the invaded cells are shown (scale bar; $100 \mu \mathrm{M}$ ). (B and D) Histograms demonstrate the miR-339-5p mRNA expression levels in the transfected cells (left), and the corresponding relative cell invasion (right); ${ }^{*} \mathrm{P}<0.05 \mathrm{vs}$. control (vector or scramble).(A and B) Followingtransfection with mimics and vector (mimics control), the expression of microRNA339-5p of A549 and H1299 increased 7 and 6 times respectively, so the number of migrating cells decreased (198_+5 vs. 56_+4, 230-+3, vs. 53+-2, P<0.05). (C and D) Followingtransfection with inhibitor and scramble (inhibitor control), the expression of microRNA339-5p of A549 decreased 2.8 times, so the number of migrating cells increased (95 +3 vs. $\left.210 \_+2, \mathrm{P}<0.05\right)$. All values presented are the mean \pm standard deviation of triplicate measurements. Experiments were repeated three times. This is one of the results. miR, microRNA. 


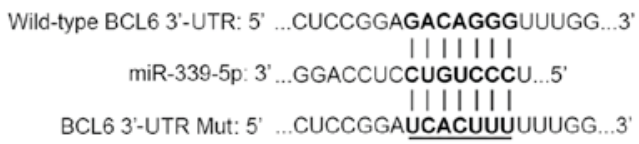

BCL6 3'-UTR Mut: 5' ...CUCCGGAUCACUUUUUUGG...3'
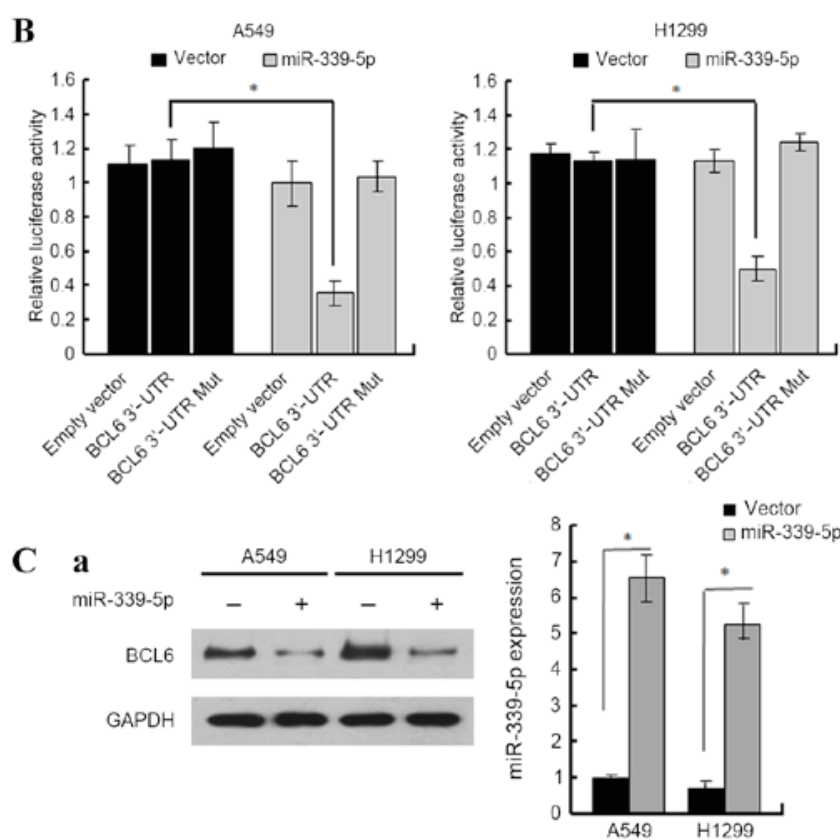

b

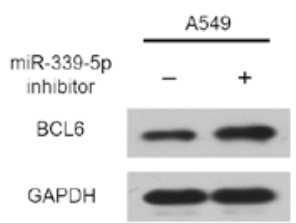

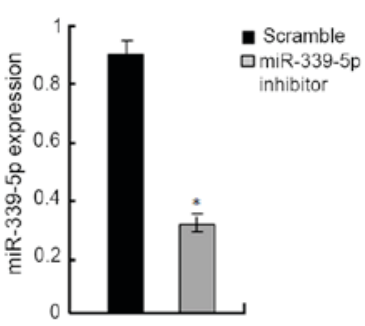

Figure 3. Inhibition of BCL6 expression by miR-339-5p in A549 and H1299 cells. (A) Wild-type and Mut forms of putative miR-339-5p target sequences (bold font) in the BCL6 3'-UTR. The underlined sequence indicates the mutated sequence. (B) Results were shown as the relative luciferase activity ( \pm standard deiviation) compared with the negative control (transfected with empty vector only) in either A549 or H1299 cells. (C) Western blot analysis of A549 and H1299 cells transfected with (a) miR-339-5p mimics or (b) miR-339-5p inhibitors. The histograms on the right demonstrate the corresponding miR-339-5p relative expression levels compared with the control (transfected with vector) in either A549 or H1299 cells for three independent experiments. All values presented are the mean \pm standard deviation of triplicate measurements. Experiments were repeated three times. " $\mathrm{P}<0.01$ Experiments were repeated three times and one results is shown. miR, microRNA; BCL6, B-cell CLL/lymphoma 6; UTR, untranslated region; Mut, mutated.

invading cells ( $\mathrm{P}<0.01$; Fig. $2 \mathrm{~A}$ and $\mathrm{B})$. For the loss-of-function assay, an inhibitor of miR-339-5p was transfected into A549 cells. Subsequently, the expression of miR-339-5p was significantly decreased, and this was concomitant with a significant increase in the number of invading cells, as compared with cells transfected with the scramble control ( $\mathrm{P}<0.01$; Fig. $2 \mathrm{C}$ and $\mathrm{D})$. These results indicate that miR-339-5p has an inhibitory effect on cell invasion.

miR-339-5p inhibits BCL6 expression by targeting its 3'-UTR. In our previous study, target prediction programs, TargetScan and miRanda, determined that $B C L 6$ was a potential target gene of miR-339-5p (11). To confirm this, Dual-Luciferase assays were performed in A549 and H1299 cell lines transfected with reporter vectors containing the wild-type or mutated forms of the BCL6 3'-UTR (Fig. 3A). The results demonstrated that transfection with miR-339-5p mimics decreased the luciferase activity in A549 and H1299 cells transfected with reporter vector containing the wild-type BCL6 3'-UTR, whereas miR-339-5p did not affect the luciferase activity in cells when the BCL6 3'-UTR was mutated (Fig. 3B). Western blot analysis demonstrated that miR-339-5p mimic transfection inhibited BCL6expression in A549 and H1299 cells compared with the controls (Fig. 3C-a). By contrast, transfection with the miR-339-5p inhibitor increased BCL6 levels in A549 cells (Fig. 3C-b). These results suggest that miR-339-5p inhibits $B C L 6$ expression by directly targeting its $3^{\prime}-\mathrm{UTR}$ in NSCLC cells.

miR-339-5p regulates the EMT by inhibiting BCL6. The EMT is one of the most important mechanisms underlying the metastatic ability of cancer cells. To determine whether miR-339-5p inhibited NSCLC cells from migrating and invading via regulation of EMT, the effects of miR-339-5p on EMT-associated markers were examined in A549 cells. The overexpression of miR-339-5p significantly inhibited the expression levels of BCL6 and the mesenchymal marker $\mathrm{N}$-cadherin, while increasing the expression of the epithelial marker E-cadherin. In addition, recovery of BCL6 expression in miR-339-5p-overexpressing cells could attenuate the effects of miR-339-5p overexpression on the regulation of EMT markers (Fig. 4A). Concordantly, downregulation of miR-339-5p by transfection with the miR-339-5p inhibitor led to increased levels of BCL6 and N-cadherin, and decreased levels of E-cadherin (Fig. 4B). These results suggest that miR-339-5p may regulate EMT, and that BCL6 is essential in this process. To verify this, miR-339-5p was overexpressed in cells in which BCL6 was knocked down, revealing that miR-339-5p overexpression failed to affect the levels of EMT markers when BCL6 was knocked down (Fig. 4C). Therefore, it was concluded that miR-339-5p regulates EMT via BCL6.

\section{Discussion}

Previously, multiple studies have demonstrated that miRNAs are associated with cancer metastasis. The molecular mechanisms underlying this association may involve various aspects, as follows.

Firstly, miRNAs can regulate the metastasis of cancer cells by regulating the expression of oncogenes or tumor suppressor genes. Asangani et al (17) identified that miR-21 stimulated invasion and migration in colon cancer cells by inhibiting programmed cell death protein 4 , which has been confirmed to be tumor suppressor gene. Additionally, Bourguignon et al (18) verified this result in breast cancer cells. Furthermore, a study by Li et al (19) demonstrated that miR-34a inhibited cell migration and invasion by the downregulation of proto-oncogene $\mathrm{C}$-met expression in human hepatocellular carcinoma cells.

Secondly, miRNAs can regulate metastasis by regulating the expression of tumor metastasis-associated genes, such as sex-determining region Y-box 4, radixin $(R D X)$ and Ras 
A

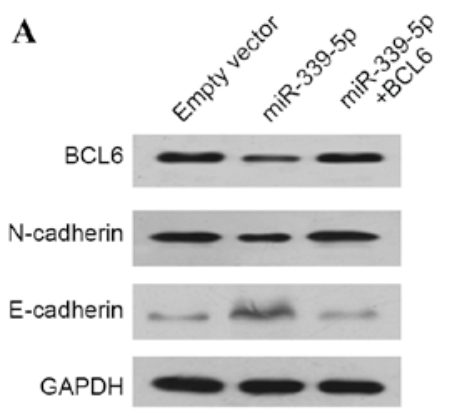

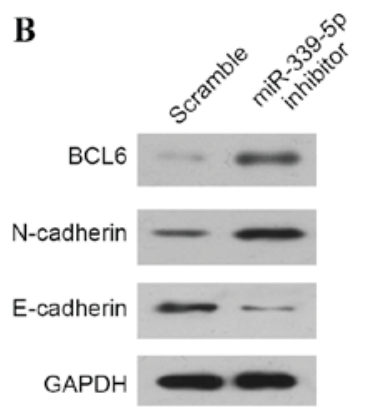

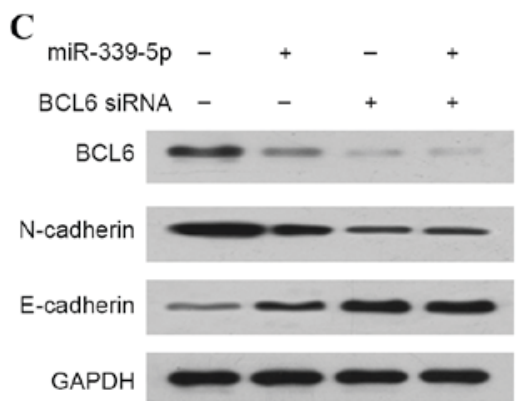

Figure 4. miR-339-5p regulates the epithelial-mesenchymal transition by inhibiting BCL6. Western blot analyses were performed on A549 cells. A549 cells transfected with (A) miR-339-5p mimics, miR-339-5p mimics plus BCL6, (B) miR-339-5p inhibitor, (C) BCL6 siRNA, or miR-339-5p mimics plus BCL6 siRNA, were cultured in regular medium. miR, microRNA; BCL6, B-cell CLL/lymphoma 6; siRNA, small interfering RNA; N-cadherin, neural cadherin; E-cadherin, epithelial cadherin.

homolog family member A (RHOA). In breast cancer cells, miR-31 inhibited cell invasion and migration by inhibiting Frizzled class receptor 3 , integrin subunit $\alpha 5$, matrix metallopeptidase 16, RDX and RHOA genes, which may promote metastasis (20). Furthermore, miR-373 and miR-520c, which are downregulated in prostate cancer cells and tumors isolated from prostatectomy compared with in their benign counterparts, may suppress cluster of differentiation 44 translation and enhance the invasion of cancer cells in vitro (21).

Thirdly, miRNAs may regulate metastasis by regulating the angiogenesis of tumor cells. Tumor growth depends on the formation of new blood vessels to facilitate blood perfusion throughout tumor tissues. A previous study showed that the downregulation of miR-130a caused the overexpression of the Homeobox A5 protein, which downregulated the expression of the angiogenesis-promoting factors vascular endothelial growth factor receptor and hypoxia-inducible factor-1, resulting in the inhibition of tumor metastasis $(22,23)$.

Fourthly, miRNAs may regulate metastasis by inhibiting DNA methylation-associated enzymes, which alter the DNA methylation status of tumor cells. Fabbri et al (24) revealed that the miR-29 family reverted aberrant methylation in lung cancer by targeting DNA methyltransferases $3 \mathrm{~A}$ and B.

Finally, miRNAs may regulate metastasis via the regulation of EMT. EMT involves the alteration from an epithelial to a fibroblast or mesenchymal cell phenotype, with cells gaining migratory ability. Numerous studies have demonstrated that EMT is important for the progress of tumor metastasis, and various miRNAs are involved in EMT regulation (25-28). The miR-200 family (which includes miR-200a, miR-200b, miR-200c, miR-141 and miR-429) are important regulators of EMT. Studies have identified that the miR-200 family could inhibit EMT and cancer cell migration by directly targeting E-cadherin transcriptional repressors, including zinc finger E-box-binding homeobox (ZEB)1, ZEB2, (also known as SIP1) and transcription factor 8 (29-31).

Our previous study suggested that miR-339-5p inhibited NSCLC cell migration and invasion; in patients with NSCLC, the expression of miR-339-5p was decreased in cancer tissues compared with normal lung tissues. Furthermore, statistical analysis demonstrated that miR-339-5p expression was inversely correlated with TNM stage and lymph node metastasis (11). In the present study, BCL6 was verified as the direct target gene of miR-339-5p, and miR-339-5p was shown to regulate EMT through BCL6.

BCL6 is a 95-kDa nuclear protein, which is encoded by a gene located on chromosome 3 q27 and acts as transcriptional repressor; it was originally characterized as a regulator of B-lymphocyte growth and development. BCL6 has been implicated in the development and growth of B-cell lymphoma (32-35). Previously, numerous studies have demonstrated that BCL6 is associated with cancer metastasis. In breast cancer cell lines, Wu et al (36) suggested that miR-339-5p may inhibit the expression of BCL6 mRNA, which was associated with suppressed migration and invasion of cells. Zhao et al (37) identified that the downregulation of miR-127 expression may promote metastasis and proliferation, and that BCL6 was a target of miR-127. Pinto et al (38) demonstrated that, in metastatic lymph nodes, the expression of BCL6 protein was significantly lower compared with that in the corresponding primary breast cancer. Hirata et al (39) revealed that maintenance of BCL6 function was important for the regulation of progression in gastric cancer. In the present study, BCL6 was confirmed to be a target gene of miR-339-5p and was demonstrated to regulate the invasiveness of NSCLC cells in vitro. Additionally, it was verified that the relative expression of peripheral miR-339-5p increases in patients with metastatic NSCLC.

In conclusion, the present study provides evidence that miR-339-5p may inhibit lung cancer cell invasion and migration by regulating EMT through BCL6 in vitro, and that miR-339-5p expression is associated with cancer metastasis, indicating that miR-339-5p may be an important biomarker for lung cancer metastasis.

\section{References}

1. Siegel R,Ma J, Zou Z and Jemal A: Cancer statistics, 2014. CA Cancer J Clinc 64: 9-29, 2014.

2. Steeq PS: Tumor metastasis: Mechanistic insights and clinical challenges. Nat Med 12: 895-904, 2006.

3. Fidler IJ: The pathogenesis of cancer metastasis: The 'seed and soil' hypothesis revisited. Nat Rev Cancer 3: 453-458, 2003.

4. Croce CM and Calin GA: miRNAs, cancer, and stem cell division. Cell 122: 6-7, 2005.

5. Iorio MV and Croce CM: MicroRNAs in cancer: Small molecules with a huge impact. J Clin Oncol 27: 5848-5856, 2009.

6. Bartel DP: MicroRNAs: Genomics, biogenesis, mechanism, and function. Cell 116: 281-297, 2004. 
7. Mendell JT: MicroRNAs: Critical regulators of development, cellular physiology and malignancy. Cell Cycle 4: 1179-1184, 2005.

8. Yanaihara N, Caplen N, Bowman E, Seike M, Kumamoto K, Yi M, Stephens RM, Okamoto A, Yokota J, Tanaka T, et al: Unique microRNA molecular profiles in lung cancer diagnosis and prognosis. Cancer Cell 9: 189-198, 2006.

9. Gibborns DL, Lin W, Creighton CJ, Rizvi ZH, Gregory PA, Goodall GJ, Thilaganathan N, Du L, Zhang Y, Pertsemlidis A and Kurie JM: Contextual extracellular cues promote tumor cell EMT and metastasis by regulating miR-200 family expression. Genes Dev 23: 2140-2151, 2009.

10. Ceppi P, Mudduluru G, Kumarswamy R, Rapa I, Scagliotti GV, Papotti M and Allgayer H: Loss of miR-200c expression induces an aggressive, invasive, and chemoresistant phenotype in non-small cell lung cancer. Mol Cancer Res 8: 1207-1216, 2010.

11. Li Y, Zhao W, Bao P, Li C, Ma XQ, Li Y and Chen LA: miR-339-5p inhibits cell migration and invasion in vitro and may be associated with the tumor-node-metastasis staging and lymph node metastasis of non-small cell lung cancer. Oncology Lett 8: 719-725, 2014

12. Kang Y and Massagué J: Epithelial-mesenchymal transitions: Twist in development and metastasis. Cell 118: 277-279, 2004.

13. Sobin LH, Gospodrowicz MK and Wittekind C (eds): International Union against Cancer: TNM Classification of Malignant Tumours. 7th edition. Wiley-Blackwell, New York, NY, 2011

14. Livak KJ and Schmittgen TD: Analysis of relative gene expression data using real time quantitative PCR and the 2(-Delta Delta C(T)) method. Methods 25: 402-408, 2001.

15. Zhang X, Zhu J, Xing R, Tie Y, Fu H, Zheng X and Yu B miR-513a-3p sensitizes human lung adenocarcinoma cells to chemotherapy by targeting GSTP1. Lung Cancer 77: 488-494, 2012.

16. Fan Z, Cui H, Xu X, Lin Z, Zhang X, Kang L, Han B, Meng J, Yan Z, Yan X and Jiao S: miR-125a suppresses tumor growth, invasion and metastasis in cervical cancer by targeting STAT3 Oncotarget 6: 25266-25280, 2015.

17. Asangani IA, Rasheed SA, Nikolova DA, Leupold JH, Colburn NH, Post S and Allgayer H: MicroRNA-21 (miR-21) post-transcriptionally downregulates tumor suppressor Pded 4 and stimulates invasion, intravasation and metastasis in colorecta cancer. Oncogene 27: 2128-2136, 2008

18. Bourguignon LY, Spevak CC, Wong G, Xia W and Gilad E: Hyaluronan-CD44 interaction with protein kinase C(epsilon) promotes oncogenic signaling by the stem cell marker Nanog and the production of microRNA-2, leading to down-regulation of the tumor suppressor protein PDCD4, anti-apoptosis, and chemotherapy resistance in breast tumor cells. J Biol Chem 284 26533-26546, 2009.

19. Li N, Fu H, Tie Y, Hu Z, Kong W, Wu Y and Zheng X: miR-34a inhibits migration and invasion by down-regulation of c-Met expression in human hepatocellular carcinoma cells. Cancer Lett 275: 44-53, 2009

20. Valastyan S, Reinhardt F, Benaich N, Calogrias D, Szász AM, Wang ZC, Brock JE, Richardson AL and Weinberg RA: A pleiotropically acting microRNA, miR-31, inhibits breast cancer metastasis. Cell 137: 1032-1046, 2009.

21. Yang K, Handorean AM and Iczkowski KA: MicroRNAs 373 and $520 \mathrm{c}$ are downregulated in prostate cancer, suppress CD44 translation and enhance invasion of prostate cancer cells in vitro. Int J Clin Exp Pathol 2: 361-369, 2009

22. Rhoads K, Arderiu G, Charboneau A, Hansen SL, Hoffman W and Boudreau N: A role for Hox A5 in regulating angiogenesis and vascular patterning. Lymphat Res Biol 3: 240-252, 2005
23. Chen Y and Gorski DH: Regulation of angiogenesis through a microRNA (miR-130a) that down-regulates antiangiogenic homeobox genes GAX and HOXA5. Blood 111: 1217-1226, 2008.

24. Fabbri M, Garzon R, Cimmino A, Liu Z, Zanesi N, Callegari E, Liu S, Alder H, Costinean S, Fernandez-Cymering C, et al: MicroRNA-29 family reverts aberrant methylation in lung cancer by targeting DNA methyltransferases $3 \mathrm{~A}$ and 3B. Proc Natl Acad Sci USA 104: 15805-15810, 2007.

25. Bracken CP, Gregory PA, Khew-Goodall Y and Goodall GJ: The role of microRNAs in metastasis and epithelial-mesenchymal transition. Cell Mol Life Sci 66: 1682-1699, 2009.

26. Gregory PA, Bracken CP, Bert AG and Goodall GJ: MicroRNAs as regulators of epithelial-mesenchymal transition. Cell Cycle 7: 3112-3118, 2008.

27. Polyak K and Weinberg RA: Transitions between epithelial and mesenchymal states: Acquisition of malignant and stem cell traits. Nat Rev Cancer 9: 265-273, 2009.

28. Ma L and Weinberg RA: Micromanagers of malignancy: Role of microRNAs in regulating metastasis. Trends Genet 24: 448-456, 2008.

29. Park SM, Gaur AB, Lengvel E and Peter ME: The miR-200 family determines the epithelial phenotype of cancer cells by targeting the E-cadherin repressors ZEB1 and ZEB2. Genes Dev 22: 894-907, 2008

30. Hurteau GJ,Carlson JA,Spivack SD and Brock GJ: Overexpression of the microRNA hsa-miR-200c leads to reduced expression of transcription factor 8 and increased expression of E-cadherin. Cancer Res 67: 7972-7976, 2007.

31. Gregory PA, Bert AG, Paterson EL, Barry SC, Tsykin A, Farshid G, Vadas MA, Khew-Goodall Y and Goodall GJ: The miR-200 family and miR-205 regulate epithelial to mesenchymal transition by targeting ZEB1 and SIP1. Nat Cell Biol 10: 593-601, 2008.

32. Chang CC, Ye BH, Chaganti RS and Dalla-Favera R: BCL-6, a $\mathrm{POZ/zinc-finger} \mathrm{protein,} \mathrm{is} \mathrm{a} \mathrm{sequence-specific} \mathrm{transcriptional}$ repressor. Proc Natl Acad Sci USA 93: 6947-6952, 1996.

33. Polo JM, Dell'Oso T, Ranuncolo SM, Cerchietti L, Beck D, Da Silva GF, Prive GG, Licht JD and Melnick A: Specific peptide interference reveals BCL6 transcriptional and oncogenic mechanisms in B-cell lymphoma cells. Nat Med 10: 1329-1335, 2004.

34. Phan RT, Saito M, Basso K, Niu H and Dalla-Favera R: BCL6 interacts with the transcription factor Miz-1 to suppress the cyclin-dependent kinase inhibitor p21 and cell cycle arrest in germinal center B cells. Nat Immunol 6: 1054-1060, 2005.

35. Jardin F, Ruminy P, Bastard C and Tilly H: The BCL6 proto-oncogene: A leading role during germinal center development and lymphomagenesis. Pathol Biol (Paris) 55: 73-83, 2007.

36. Wu ZS, Wu Q, Wang CQ, Wang XN, Wang Y, Zhao JJ, Mao SS, Zhang GH, Zhang N and Xu XC: miR-339-5p inhibits breast cancer cell migration and invasion in vitro and may be a potential biomarker for breast cancer prognosis. BMC Cancer 10: 542, 2010.

37. Zhao X, Duan Z, Liu X, Wang B, Wang X, He J, Yao Z and Yang J: MicroRNA-127 is downregulated by Tudor-SN protein and contributes to metastasis and proliferation in breast cancer cell line MDA-MB-231. Anat Rec (Hoboken) 296: 1842-1849, 2013.

38. Pinto AE, André S, Silva G, Vieira S, Santos AC, Dias S and Soares J: BCL-6 oncoprotein in breast cancer: Loss of expression in disease progression. Pathobiology 76: 235-242, 2009.

39. Hirata Y, Ogasawara N, Sasaki M, Mizushima T, Shimura T, Mizoshita T, Mori Y, Kubota E, Wada T, Tanida S, et al: BCL6 degradation caused by the interaction with the C-terminus of pro-HB-EGF induces cyclin D2 expression in gastric cancers. Br J Cancer 100: 1320-1329, 2009. 